\title{
The Production of the Opposite Adjectives in the Language of the Yemeni Children with Autism
}

\author{
Tahani A.R. Al-absi \\ Ph.D. Researcher in Linguistics
}

\begin{abstract}
Aims: The main goal of the present study is to shed the light on the production of the opposite adjectives in the language of the Yemeni autistic children. Also, it compared the performance of the autistic children to the performances of typically developing children matched them chronologically and mentally. Methods \& Procedures: A group of children with autism aged 7 years old chronologically and 4 years old mentally, matched to a control group of typically developing children aged 7 years old chronologically, and to a control group of typically developing children aged 4 years old mentally. The examiner used the Expressive Language Task to elicit the opposite adjectives from the three participated groups of children. Results: The findings of this study reported the significant difference between the performance of the autistic children on the adjectives, and the control TD groups of children who matched them chronologically and mentally. Additionally, the features of the opposite adjectives in the language of the autistic children were included in the current study based on their performance.Conclusion and Implication: The children with autism may face more difficulties in producing the opposite adjectives in their language. Particularly, the adjective which involve understanding the nonverbal language (e.g. happy \& sad), the adjectives which they don't use regularly in their daily activities (e.g. full \& empty) or the adjectives which can be confused with other adjectives such as confusing (tall $\&$ short or thin $\&$ fat) with adjectives such as (big \& small). Further, the children with autism don't acquire and learn their mother tongue spontaneously as the typically developing children. Therefore, they need extensive teaching and effective teaching methods and tools to learn the opposite adjectives more extensively and use them properly.
\end{abstract}

Keywords: autism, production, adjectives, language, features.

DOI: $10.7176 /$ JLLL/76-05

Publication date: February $28^{\text {th }} 2021$

\section{Introduction}

Autism is a complex neurodevelopmental disorder causes impairments in the social and communication skills of the autistics, in addition to restricted repetitive and stereotyped behaviors and activities (APA, 1994). The symptoms of autism range from mild to severe, and every autistic individual is different from the others and with unique symptoms (APA, 2013). The language in the children with autism characterized with delay or no spoken language, use the language literally without any changes to suit the new situation and context, reversing the pronouns (e.g., using the pronoun (you) instead of the pronoun (I), the repetition of the phrases, sentences and questions, using language but not for the purpose of communication (Leo Kanner,1943). The children with autism have impairments in the nonverbal communicative behaviors which used in the social communication with the others, and included abnormalities in understanding the facial expressions of the others (APA, 2013), therefore, such difficulties resulted in their impaired comprehension and production of adjectives such as (happy and sad) in examples such as (happy face) \& (sad face).

In the Modern Standard Arabic, Adjectives inflect to gender (masculine or feminine), number (singular or plural), case (nominative, accusative or genitive), definiteness (definite or indefinite) and degree (comparative and superlative) (Karin C.Ryding, 2005\&Karin C.Ryding, David J. Mehall, 2005). In Arabic, Adjectives have base forms and can be inflected to masculine or feminine singular adjectives to specify the masculine or feminine singular nouns, or the masculine or feminine dual nouns, or the masculine or feminine plural nouns (Karin C.Ryding, 2005).

This table illustrates Arabic adjectives inflected to gender and number in Arabic language:

\begin{tabular}{|c|c|c|c|c|c|}
\hline \multicolumn{3}{|c|}{ Gender \& Number } \\
\hline \multirow{2}{*}{ Adjective } & \multirow{2}{*}{ Gender } & \multicolumn{3}{|c|}{ Number } & Peaning in English \\
\cline { 3 - 5 } & & Singular & Dual & Plural & \\
\hline Jamil & Masculine & Jamil & Jamilan & Jamileen & Handsome \\
\hline jamila & Feminine & Jamila & Jamilatan & Jamilat & Beautiful \\
\hline
\end{tabular}

\section{Literature Review: -}

To my knowledge, there are no Arabic previous studies about the production of adjectives in the language of the Arab autistic children. Moreover, there are no Yemeni previous studies about the production of adjectives in the language of the Yemeni autistic and TD children who speak Yemeni Arabic. Moreover, there are no studies in 
English focused on the production of adjectives in the language of the autistic children or investigated this issue separately. Therefore, I conducted this study to shed the light on this issue and included the few available previous studies which examined the adjectives in the language of children.

Durrleman \& Zufferey (2009) conducted a study to explore the syntactic structures in the language of the children with autism, and indicated that the two participants of the study used the adjectives frequently in their productions. Moreover, the finding of the study stated that the upper layers of the syntactic tree are impaired more than the lower layers, and the position of the adjectives is in the low layers of the syntactic tree as stated by Cinques (2000). Therefore, they concluded that the adjectives are used frequently in the language of the children with autism, and they faced no difficulties in using them as the finding of their study reported.

Cassidy, B. (1980) examined the relationship between the comprehension of the dimensional adjectives (e.g., long-short, high-low) and the chronological age. The sample of this study was 70 TD children aged between 2;2 and 8;2 years and divided into 7 age groups. All of them were selected based on their age, normal hearing and level of vocabulary. The findings of this study stated that there was a relationship between the comprehension of the adjectives and age as the ration of the adjectives increased in the production of the participants with age. Moreover, there was inconsiderable difference in their understanding of the unmarked adjective and its versus marked adjectives except for three years old age group.

Miranda-Linne, F. \& Melin, L. (1992) investigated the possibility of teaching two autistic children to produce two color adjectives in their expressive language when they describe their favorite types of food and toys. They used the traditional discrete-trial procedures and the incidental teaching in teaching them how to use these twocolor adjectives. The teaching was in the class but the generalization of the adjectives and using them spontaneously assessed by parents at home. The findings of this study revealed the efficiency of the traditional discrete-trail procedures in teaching the autistic children and resulted in their fast acquisition and great generalization of the adjectives. Moreover, the using of the incidental teaching method in teaching them resulted in storing these adjectives in their memory, in addition to great generalization and spontaneous usage of these adjectives in their expressive language. Even though, the incidental teaching method requires longer time to help them in acquiring language but affective and recommended in teaching the autistic children as it may results in permanent knowledge.

Reis, R. \&Antonio, T. (2012) investigated the morphosyntactic classes in the language of the children with autism. The researcher recorded the speech of 4 children with autism aged between 3 and 13 years during their activities, and their degree of autism ranged from mild to severe. The findings of this study stated that the verbs and nouns were used more than any other classes in their production, in opposite to the Adjectives which used less frequently. Moreover, they avoided the long and complex syntactic structures in their production.

\subsection{Research Questions: -}

1) What is the significant difference between the production of the opposite adjectives in the language of the Yemeni children with autism and the control groups of the typically developing children who matched them chronologically and mentally?

2) What are the features of adjectives in the language of the Yemeni children with autism based on their performance in the study?

\subsection{Purpose of the study}

The primary goal of the current study is to investigate the opposite adjectives in the language of the children with autism. Also, it compares their performance to the performances of a group of typically developing children matched them on the chronological age, and another group of the typically developing children matched them on the mental age. Besides, the findings of the present study shed the light on the difficulties which face the autistic children in using these adjectives in their production of language in comparison to TD groups of children matched them chronologically and mentally. Moreover, the findings of this study could be used as a guide for the teachers and parents of the autistic children to focus on the difficulties which face these children in comprehending and producing the opposite adjectives properly and easily in the production of their language. Furthermore, the findings of this study could be used as a guide for designing more appropriate curriculums, teaching tools and methods help the children with autism to overcome the difficulties which face them in producing the adjectives.

\subsection{Samples/Participants}

The sample of this study consisted of 20 children with high functioning autism aged 7 years old chronologically and 4 years old mentally, matched to a group of 20 Typically Developing Children on the chronological age, and 20 Typically Developing Children on the mental age. The autistic children were diagnosed based on the criteria of DSM-IV. The children with autism had no hearing or vision difficulties, and the Typically Developing children had no neurological disorders. The children with autism were able of producing three or four words 
sentences, but haven't received a good teaching in the autism centers or at home due to various reasons. On the other hand, they were able of overcoming their deficits of language with the help of appropriate teaching tools, methods and qualified teachers. The autistic children were visited in their homes and the Typically Developing children were visited in their homes or kindergarten in Sana'a-Yemen.

\subsection{Instruments}

The materials of this study were 6 pictures represented two adjectives reversing each other (happy vs. sad), (hot vs. cold), (tall vs. short), (big vs. small), (fat vs. thin) and (full vs. empty). The children were required to produce the adjective which reverses the one uttered by the examiner in a sentence such as (this strawberry is big, what about the other one?)
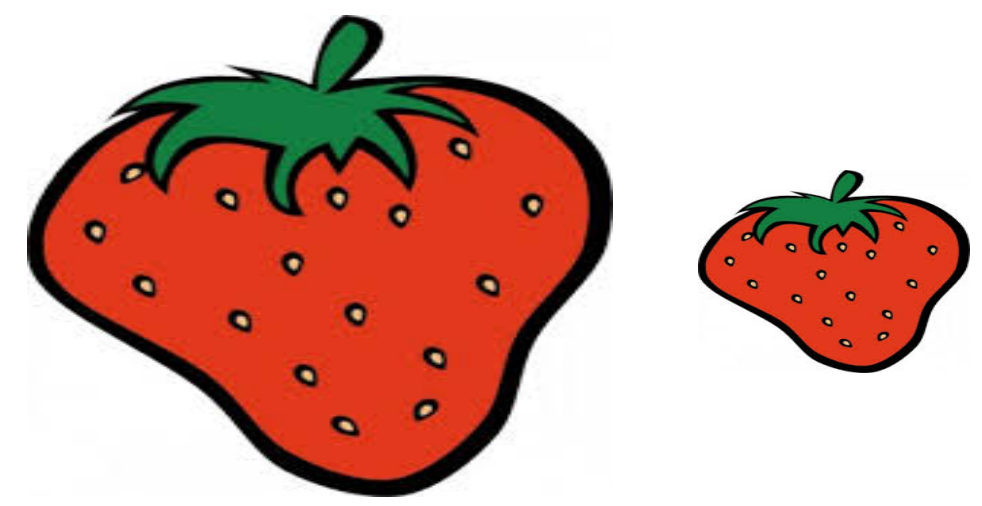

picture (1) illustrates the opposite adjectives (big \& small) and used in the current study

\subsection{Data collection procedures}

The examiner used the Expressive Language Task to collect the data from the three participated groups of children. In this task, each paper included two pictures portrayed two adjectives reversing each other. The examiner placed the papers in front of the children one by one, read a sentence included one of the adjectives, and asked about the opposite adjective in a question (e.g., this strawberry is big, what about the other one?) and the participant was required to produce the opposite adjective in his answer to be scored.

\section{Results}

Table (1) Illustrates the Mean Ranks of the Groups' Performances on the Adjectives according to KruskalWallis Test: -

\begin{tabular}{|c|c|c|c|}
\hline No. & Type of Adjectives & Groups & Mean Ranks \\
\hline \multirow{3}{*}{1} & \multirow{3}{*}{ Happy vs. Sad } & Children with Autism & 21.50 \\
\hline & & TD group of chronological age control & 39.50 \\
\hline & & TD group of mental age control & 30.50 \\
\hline \multirow{3}{*}{2} & \multirow{3}{*}{ Hot vs. Cold } & Children with Autism & 26.00 \\
\hline & & TD group of chronological age control & 35.00 \\
\hline & & TD group of mental age control & 30.50 \\
\hline \multirow{3}{*}{3} & \multirow{3}{*}{ Tall vs. Short } & Children with Autism & 23.50 \\
\hline & & TD group of chronological age control & 38.50 \\
\hline & & TD group of mental age control & 29.50 \\
\hline \multirow{3}{*}{4} & \multirow{3}{*}{ Big vs. Small } & Children with Autism & 28.50 \\
\hline & & TD group of chronological age control & 33.00 \\
\hline & & TD group of mental age control & 30.00 \\
\hline \multirow{3}{*}{5} & \multirow{3}{*}{ Thin vs. Fat } & Children with Autism & 23.00 \\
\hline & & TD group of chronological age control & 39.50 \\
\hline & & TD group of mental age control & 29.00 \\
\hline \multirow{3}{*}{6} & \multirow{3}{*}{ Full vs. Empty } & Children with Autism & 21.50 \\
\hline & & TD group of chronological age control & 41.00 \\
\hline & & TD group of mental age control & 29.00 \\
\hline
\end{tabular}




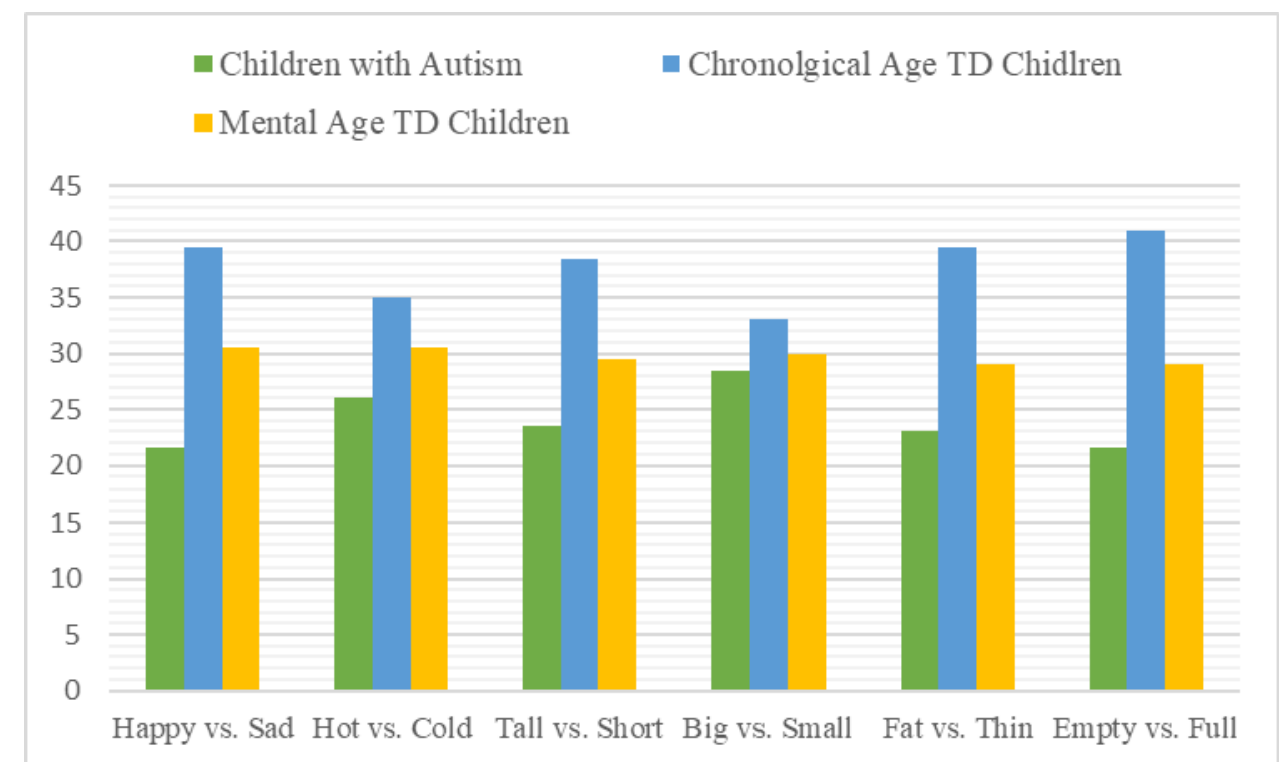

Diagram (1) Illustrate the Mean Ranks of the Groups' Performances on the Opposite Adjectives

According to the results of Kruskal-Wallis tests, there were no significant differences between the groups' performances on the adjectives (hot vs. cold) and (big vs. small). Further, the result of Kruskal-Wallis test for the adjectives (hot vs. cold) was $(\mathrm{H}(2)=5.531, \mathrm{P}>0.05)$ and $(\mathrm{H}(2)=1.986, \mathrm{P}>0.05)$ for the adjectives (big vs. small).

Regarding the adjectives (happy vs. sad), there was a significant difference between the groups' performances as indicated by Kruskal-Wallis test $(\mathrm{H}(2)=15.560, \mathrm{P}<.01)$. As a result, post hoc tests were conducted to find out the significant differences between the performances of the participated groups. The results of the pairwise comparisons indicated that there was a significant difference between the performances of the autistic children and the TD group of chronological age control as the autistic children (Median= .00, $\mathrm{n}=20$ ) and the TD group of chronological age control (Median=1.00, $\mathrm{n}=20$ ), $\mathrm{U}=80.000, \mathrm{Z}=-3.928, \mathrm{p}<.01, \mathrm{r}=0.1$. Moreover, there was no significant differences between the performances of the TD group of chronological age control and the TD group of mental age control or between the performances of the Autistic group of children and the TD group of mental age control as $\mathrm{p}>0.05$.

There was a significant difference between the groups' performances on the adjectives (tall vs. short) as the autistic children replaced these two adjectives with the adjectives (big vs. small) most of the times. Therefore, the results of Kruskal-Wallis tests showed that $(\mathrm{H}(2)=11.512, \mathrm{P}<0.05)$. Accordingly, post hoc tests were executed to find out the significant difference between the groups' performances on the adjectives (tall vs. short). The results noted that there was a significant difference between the performances of the autistic children (Median $=.00, \mathrm{n}=20$ ) and the TD group of chronological age control (Median=1.00, $\mathrm{n}=20), U=100.000, Z=-$ $3.407, \mathrm{p}<.01, \mathrm{r}=0.1$. Also, there was no significant difference between the performances of the TD group of mental age control and the TD group of chronological age control or between the performances of the children with autism and the TD group of mental age control.

According to Kruskal-Wallis test, there was a significant difference between the performances of the three participated groups on the adjectives (fat vs. thin) as $(\mathrm{H}(2)=13.399, \mathrm{P}<0.05)$. Thus, post hoc tests were performed to find out the significant difference between the groups' performances. The results of the tests showed that there was a significant difference between the performances of the autistic children (Median $=.00$, $\mathrm{n}=20$ ) and the TD group of chronological age control (Median $=1.00, \mathrm{n}=20$ ), $U=90.000, Z=-3.667, \mathrm{p}<.01$, $\mathrm{r}=0.1$. In addition, there was no significant difference between the performances of the autistic children and their TD group of mental age control or between the performances of the TD group of mental age control and the TD group of chronological age control.

Kruskal-Wallis test was used to check the performances of the three groups on the adjectives (empty vs. full). The results indicated that there was a significant difference between their performances as $(H(2)=17.618$, $\mathrm{P}<.01)$. Consequently, post hoc tests were conducted to find out the significant difference between the groups' performances. The results indicated that there was a significant difference between the performances of the autistic children (Median=00., $\mathrm{n}=20$ ) and the TD group of chronological age control (Median=1.00, $n=20$ ), $U=70.000, Z=-4.192, \mathrm{p}<.01, \mathrm{r}=0.1$. Besides, there was a significant difference between the performances of the TD group of chronological age control (Median=1.00, $n=20$ ) and the TD group of mental age control (Median=1.00, $\mathrm{n}=20), U=120.000, Z=-2.884, \mathrm{p}<0.05, \mathrm{r}=0.1$. Further, there was no significant difference between the performance of the autistic children and the TD group of mental age control. 


\section{Discussion}

The autistic children used the adjectives (big $\&$ small) easily but couldn't use them properly since in Arabic the adjectives inflect to gender (masculine or feminine), and there was no agreement in their production between these two adjectives and the nouns which preceded them in case of the feminine nouns. Therefore, they omitted the feminine suffixes ( $\mathbf{T}$ (/tā' marbūTa) and replaced it with its masculine suffixes. Thus, they replaced the feminine adjective (kabeeratun) with the masculine adjective (kabeerun) and the feminine adjective (sagheeratun) with the masculine adjective (sagheerun). On the other hand, the TD groups of chronological and mental age control comprehended and produced them easily without any difficulties, and inflected the adjectives to its feminine gender properly. Sometimes, the TD group of mental age control used the adjectives (big\&small) in describing adjectives such as (long \& short) or (fat \& thin) in case these adjectives aren't used frequently in their environment, due to factors such as late schooling and poor social interaction. Furthermore, the autistic children used the same techniques to avoid their poor knowledge of the adjectives (long \& short) or (fat \&thin), due to factors such as late spoken language, poor social interaction, bad teaching techniques and tools in some of the autism centers in Yemen.

Most of the autistic children used the adjectives (hot\&cold) easily and realized them without any difficulties, as these two adjectives are common and used frequently in their daily activities at home. On the other hand, there was no agreement between these adjectives and the nouns which preceded them in case of describing feminine nouns, as a result they used them easily but not properly. Furthermore, the TD groups of chronological and mental age control used them easily and properly without any difficulties.

The autistic children and the TD group of mental age control couldn't use the adjectives (full\&empty) properly and replaced them with description and negation. They described the full basket with sentences such as (there are strawberries in this basket), and described the empty basket with negation answers such as (there are no strawberries in the other basket). In opposite to, the TD group of chronological age control which described these two adjectives properly.

The autistic children couldn't use the adjectives (tall\&short) properly and replaced them with the adjectives (big \& small) most of the times, whereas the performance of the TD group of mental age control was better, but some of them replaced the adjectives (tall \& short) with the adjectives (big \& small) as well. Further, the two groups used the adjective (big) to describe the (tall man), and the adjective (small) to describe the (short man). Whereas, the TD group of chronological age control used them easily and properly without any difficulties.

The autistic children couldn't use the adjectives (fat $\&$ thin) properly and replaced them with the adjectives (big \& small). They described the fat person as (big), and the thin person as (small) because (big and small) are common adjectives and used frequently in their daily speech. In addition, these two adjectives are similar in size to (fat \& thin). Also, some of the TD group of mental age control used them as the autistic children, and replaced them with the adjectives (big \& small). Besides, some of the autistic children and the TD group of mental age control used the negation (not fat), when I asked them (if this man is fat, what about the other one?), and the replies were negated phrases (e.g. not fat). Moreover, the TD group of chronological age control used them easily and properly without any difficulties.

The autistic children couldn't use the adjectives (happy \& sad) properly and replaced them with verbs. They described the happy face with the verb (smile), and the sad face with the verb (cry). Moreover, the performance of the TD group of mental age control was better than the performance of the autistic children, but some of them replaced these two adjectives with the verbs (smile \& cry), due to reason such as the lack of the social interaction or late schooling. In contrast to, the TD group of chronological age control who had no problems with comprehending and producing the facial expressions (e.g. happy\&sad) and the non-verbal language. Whereas, the autistic children failed to comprehend and produce these two adjectives easily, as result to their impaired non-verbal language which include the facial expressions.

The TD group of chronological age control were able of comprehending and producing the adjectives properly and easily, due to their early acquisition of the spoken language, and their spontaneous learning and producing of the adjectives. Unlike, the group of the children with autism who matched them on age chronologically, but acquired the spoken language at late age, and it reflected on their comprehension and production of the adjectives. Moreover, the cognitive abilities of the children with autism affected the processes of learning and producing these adjectives spontaneously. On the other hand, there are similarities between the performances of the children with autism, and the TD group of mental age control who aged 4 years old, and matched them mentally, as they were still young TD children, and exposed limitedly to the various types of adjective.

Additionally, the current study emphasized that there is a correlation between their knowledge of the various adjectives and age, as the TD group of the chronological age control were able of comprehending and producing the adjectives properly and easily. Differing from, the TD group of the mental age control who performed less than them, due to their young age and limited exposure to the various adjectives, but better than the children with autism. Furthermore, these findings agree with the findings of Cassidy B.'s (1980) study which 
indicated the correlation between the increase of the adjectives in the language of the children and their progress in age. Furthermore, the findings of this study aren't consistent with the findings of Durrleman et. al.'s (2009) study which stated that the autistic children of their study used the adjectives frequently in their production. On the other hand, the findings of the current study are in line with the findings of Reis et. al.'s (2012) which revealed that the autistic children of their study used the adjectives less frequently in their production.

The findings of this study specified the features of using the opposite adjectives in the language of the children with autism based on their performance and included:

1) The autistic children face difficulties with maintaining the agreement between the adjectives and the nouns which precede them in case of describing feminine nouns, as they replace the feminine suffixes (T (/tā' marbūTa) with masculine suffixes.

2) The autistic children have a good knowledge of the adjectives which they use frequently in their daily activities at home or in their surrounding in general (e.g. big and small).

3) The autistic children face difficulties with comprehending and producing the adjectives of facial expressions (e.g. happy \& sad) due to their lack or impaired non-verbal language.

4) The autistic children replace some adjectives with verbs. For example, replaced (cry \& smile) with (happy \& sad) to avoid the difficulties which face them in understating the facial expressions and the non-verbal language.

5) The autistic children use the description instead of the adjectives when they aren't familiar with the adjectives. For example, they replaced (a full basket) with (there are strawberries in the basket) and (an empty basket) with (there are no strawberries in the basket).

6) The autistic children use the negation instead of the adjectives when they aren't familiar with the adjectives. For example, they replaced the adjective (thin) with the negation (not fat).

7) The children with autism replace the adjectives which they have no knowledge about with the adjectives which they use frequently in their daily life. For example, they replaced the adjective (tall) with (big) and the adjective (short) with (small).

\section{Conclusion}

To sum up, the performances of the TD group of the chronological age control reached the ceiling on all the adjectives, in contrast to the other groups which performed less than them due to the impaired cognitive functioning in the children with autism, and the young age in the TD group of mental age control. The performances of the autistic children, and the TD group of mental age control on the adjectives (big vs. small), and the adjectives (hot vs. cold) were good, but their performances on the rest of the adjectives (happy vs. sad \& fat vs. thin \& tall vs. short) were similar and less than the other adjectives. The individuals with weak central coherence as in the children with autism concentrate on the details more than on the general meaning due to their concentration on small information rather than on the general information (Happe\&Frith,2006). Therefore, the children with autism replaced the adjectives with description of the details, negation, and verbs instead of using the general information such as the adjectives, and it was attributed to their weak central coherence.

\section{References}

American Psychiatric Association, APA (1994). Diagnostic and Statistical Manual of Mental Disorders, fourth edition: DSM-IV. APA, Washington.

American Psychiatric Association APA (2013). Diagnostic and Statistical Manual of Mental Disorders, fifth edition: DSM-IV. APA, Washington DC.

Cassidy, B. (1980). "Age and the comprehension of spatial adjectives". Dissertations and theses. https://doi.org/10.15760/etd.2960.

Happe, F.,\& Frith, U.(2006). The weak coherence account: Detail-focused cognitive style in autism spectrum disorders. Journal of autism and development disorders, 36(1),5-25.

Kanner, L. (1943). "Autistic disturbances of affective contact". Nervous Child, 2, 217-250.

Miranda-Linne, F. \& Melin, L. (1992). Acquisition, generalization, and spontaneous use of color adjectives: a comparison of incidental teaching and traditional discrete-trial procedures for children with autism. Res Dev Disabil.13(3):191-210. doi:10.1016/0891-4222(92)90025-2.

Reis, R. \& Antonio, T. (2012). Morphosyntactic Analysis of Language in Children with Autism Spectrum Disorder. doi//:10.1007/978-3-642-28885-2 4.

Ryding, C. K. (2005). A Reference Grammar of Modern Standard Arabic. UK, Cambridge: Cambridge University Press.

Ryding, K. \& Mehall, D. (2005). Formal Spoken Arabic (Basic Course with MP3 Files). USA. Washington, D.C. Georgetown university press. 